\title{
Malignant primary nerve sheath tumor of the cauda equina in a patient without Von Recklinghausen disease
}

\author{
Amit Shankar Singh, Virendra Atam, Liza Das, Mallagouda Patil, Besthenahalli Erappa Yathish, \\ Sciddhartha Kunwar, Ganesh Seth \\ Department of Medicine, King George's Medical University, Lucknow, Uttar Pradesh, India
}

\section{A B S T R A C T}

Malignant peripheral nerve sheath tumor (MPNST) is a rare variety of soft-tissue sarcoma of ecto-mesenchymal origin. Various sites have been mentioned, but it is rare in cauda equina. Only five cases of MPNST of cauda equina are reported till date. A 42-year-old male presented with complaints of weakness in lower limbs for last 5 months with bladder and bowel dysfunction. On magnetic resonance imaging, an isointense lesion on T1 and a hyperintense on T2 imaging was found at terminal spinal cord level. The lesion was excised following laminectomy and showed varied cellularity with thin oval, polyhedral and spindle-shaped cells arranged in bundles on microscopic examination suggesting MPNST. MPNST of cauda equina is rare and is associated with Neurofibromatosis Type-1 or radiation exposure, but is rarer without these risk factors. Prognosis is favorable in these cases.

Key words: Nerve sheath tumors, Von Recklinghausen disease, cauda equina, laminectomy

\section{INTRODUCTION}

Malignant peripheral nerve sheath tumor (MPNST) is a rare variety of soft tissue sarcoma of ecto-mesenchymal origin with estimated overall incidence in the general population of approximately $0.001 \% .^{[1,2]}$ Several sites of MPNST are described usually peripheral nerves of the trunk, but its occurrence in cauda equina is rare. ${ }^{[3]}$ World Health Organization coined the term malignant primary nerve sheath tumor replacing previous heterogeneous and often confusing terminology, such as malignant schwannoma, malignant neurilemmoma, and neurofibrosarcoma, for tumors of neurogenic origin and similar biological behavior. ${ }^{[4]}$ They arise from a major or minor peripheral nerve branches or sheath of peripheral nerve fibers. These tumors may arise spontaneously in adult patients, although $5-42 \%$ of MPNST have an association with neurofibromatosis Type-I (Von Recklinghausen disease)..$^{[5]}$

Recently Xu et al. enumerated 23 cases of intradural MPNST located in different parts of the spine. ${ }^{[6]}$

\begin{tabular}{|l|l|}
\hline \multicolumn{2}{|c|}{ Access this article online } \\
\hline Quick Response Code: & Website: \\
\hline & www.ijns.in \\
\cline { 2 - 2 } & \\
\hline
\end{tabular}

However, only five case of MPNST of cauda equina had been reported in literature ${ }^{[7-11]}$ all over the world and only one case in India. ${ }^{[7]}$ We are reporting the sixth case in the literature from our center.

\section{CASE REPORT}

A 42-year-old otherwise healthy male, carpenter by occupation presented to our department with complaints of weakness in both lower limbs of 5 months duration. Initially weakness started from right lower limb, and then progressed to left lower limb over a period of 1 month. The patient also complained of tingling sensations around perianal area for the last 3 months. He developed bladder and bowel dysfunction for the last 3 weeks. Patient was bed ridden for last 1 month, and was unable to do his routine works. There was no history of any trauma to back, subcutaneous nodules on skin or radiation exposure in the past.

On examination, decreased power was found in both lower limbs along with increased reflexes. Sensations were diminished in the perianal area and a bedsore was present in the sacral area of the patient.

Patient underwent magnetic resonance imaging (MRI) of lumbosacral region, which revealed a lesion with non-uniform isointense signal on T1-weighted and a heterogenously hyperintense lesion extending from L1 to L4 level in T2-weighted images. Gadolinium-enhanced

Address for correspondence: Dr. Amit Shankar Singh,

Department of Medicine, King George's Medical University, Lucknow, Uttar Pradesh, India. E-mail: amitkgmumedicine@gmail.com 
MRI revealed enhancement of the tumor except in multiple foci of high intensity [Figures 1 and 2].

To alleviate these symptoms he underwent L1 to L5 laminectomy along with excision of nodular lesion attached to lumbar and sacral nerve roots. The excised lesions were sent for histopathological analysis, which showed varied cellularity with thin oval, polyhedral and spindle-shaped cells arranged in bundles on microscopic examination. The cells were pleomorphic and arranged without a specific pattern. Most cell nuclei were swollen and showed a moderate amount of chromatin with cigar shaped nuclei along with dispersed pyknosis and karyorrhexis in few cells [Figure 3].

Following confirmation of malignant nerve sheath tumor patient also received radiation therapy. With surgery and radiation therapy, power of lower limbs and bladder bowel dysfunction improved gradually within 2 months of therapy.

\section{DISCUSSION}

MPNST of cauda equina are rare sarcomas, which originate from peripheral nerves or from cells associated with the nerve sheath, such as Schwann cells, perineural cells, or fibroblasts. Only five cases of MPNST of cauda equina are reported till date, and we are describing the sixth.

About $60-80 \%$ of nerve-sheath tumors arise from nerve roots before leaving the dural sac. A further 10\% arise

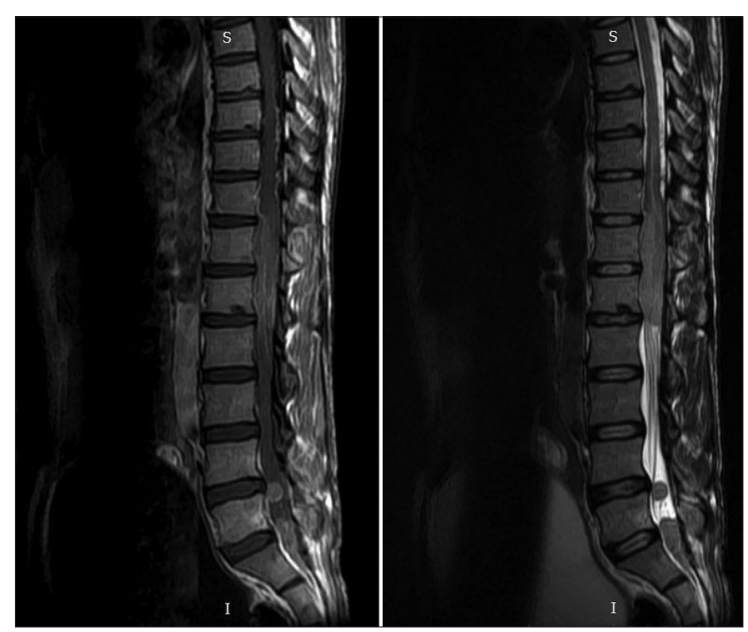

Figure 1: T1-weighted fast spin echo sequence sagittal view image of spinal magnetic resonance imaging revealed a lesion extending from terminal part of spinal cord to conus medularis with non-uniform isointense signal with contrast enhancement along with widening of spinal canal and T2-weighted spin echo sequence sagittal view showing heterogenously hyperintense signal of lesion of terminal spinal cord. Also section showing round homogenous intensities along the nerve roots as the nerve root leaves the dural sac and becomes surrounded by the dural-root sleeve. These tumors; therefore may display both intradural and extradural components (dumbbell tumor). Nerve sheath tumors that are entirely extradural or intramedullary are less common. Intradural nerve sheath tumors most commonly affect the lumbosacral region, but cervical and thoracic tumors have been reported too. Intradural nerve-sheath tumors might be more common in the lumbosacral region because of the longer intradural segment of the caudal spinal nerve roots in the neuraxis. Nerve-sheath tumors are generally regarded as benign neoplasms, but can be malignant in a few cases, where they are designated the term malignant peripheral nerve-sheath tumors. Although more than $50 \%$ of MPNST are associated with neurofibromatosis Type-1, only a small percentage of patients with this disease have malignant neoplasms.

The etiology is unknown, but there is a higher incidence in patients with a history of radiation exposure ${ }^{[8]}$ or a preexisting neurofibroma. Cross sectional studies have previously demonstrated a $1-2 \%$ prevalence of MPNST among NF1 patients, but another study showed these patients have a $10 \%$ lifetime risk of ultimately developing an MPNST. The development of plexiform neurofibromas has been linked to the loss of NF1 gene expression in a mouse model while the development of MPNST has been related to other genetic insults, such as those involving p53 and p16. ${ }^{[12]}$ While NF1 gene activity does not independently cause MPNSTs, it may in fact predispose these patients to such an event.

MPNSTs generally occur in adulthood, typically between the ages of 20 and 50 years of age. Approximately, 10-20\% of cases have been reported to occur in the first 2 decade

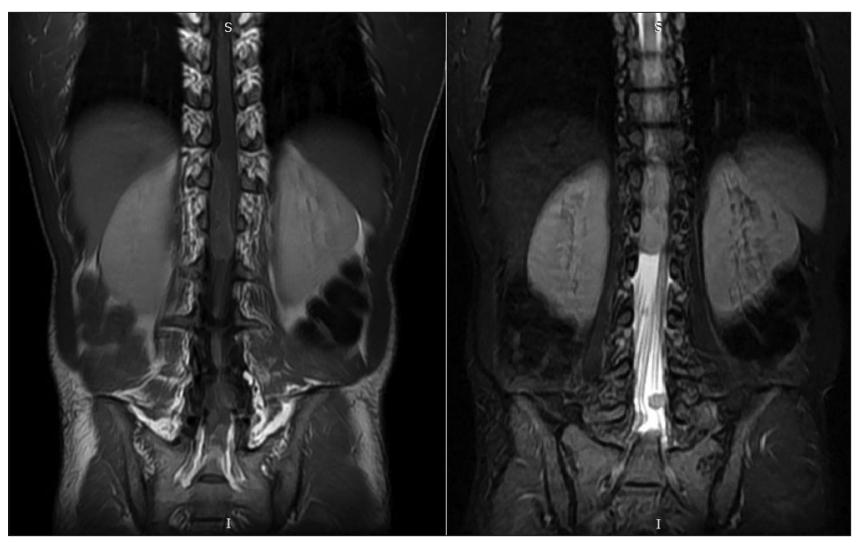

Figure 2: T1-weighted fast spin echo sequence coronal view image of spinal magnetic resonance imaging revealed a lesion extending from terminal part of spinal cord to conus medularis with non-uniform isointense signal and coronal short tau inversion-recovery image showed a hyperintense signal lesion of terminal spinal cord with round homogenous hyperintensities along nerve roots 


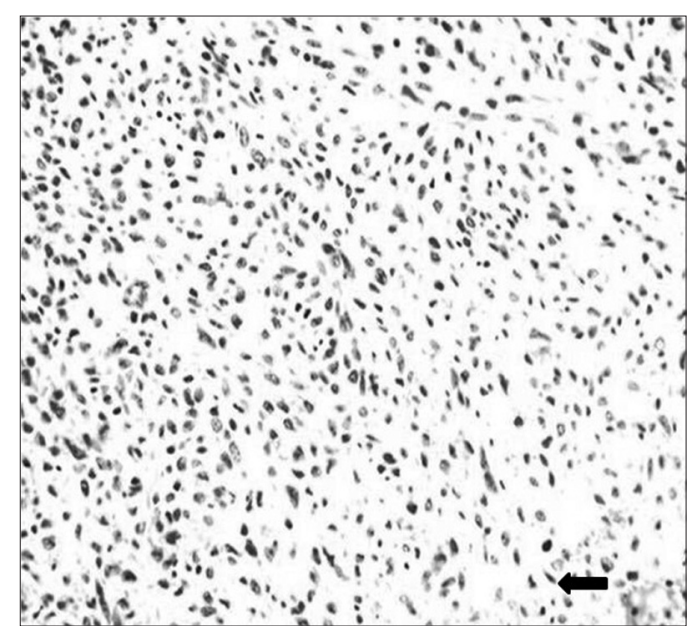

Figure 3: Intraoperative histology sections on microscopic examination showing varied cellularity with thin oval, polyhedral and spindle-shaped cells arranged in bundles

of life, with occasional cases involving infants as young as 11 months of age. These tumors occur with equal frequency in males and females and some series have shown a female preponderance.

The following criteria were used for the histological diagnosis of MPNST (a) gross fusiform tumors in relation to nerves, (b) microscopic features of spindle cell with fascicular pattern and varying degrees of mitosis, necrosis and tumor calcification, (c) presence of associated benign neurofibroma or schwannanian cells, and (d) positive immunohistochemical staining for S-100 protein, neuron specific enolase and others like actin, cytokeratin, smooth muscle actions, desmin, and vimentin to differentiate from other spindle cell sarcomas. The tumors were classified as low and high grade on the basis of their cellular differentiation, mitotic count, tumor necrosis and expression of MIB-1 proliferation marker. ${ }^{[13]}$ Tumor necrosis was evaluated with scoring as 0,1 , and 2 , depending on the percentage of necrosis as $0 \%,<50 \%$, and $>50 \%$ presence, respectively. Unusual features of MPNST include heterologous mesenchymal and epithelial elements, as well as the occurrence at sites of previous irradiation, i.e., post-radiation sarcornas. ${ }^{[4]}$ In general, the prognosis of MPNST is grim, and survival is reportedly influenced by tumor location and size and association with von Recklinghausen's disease. ${ }^{[14]} \mathrm{On}$ MRI nerve-sheath tumors have an isointense signal on T1-weighted images and a hyperintense signal on T2-weighted images. Gadolinium adds variable enhancement ranging from a homogeneous to a peripheral ring-like enhancement.
Treatment for MPNST of cauda equina is similar to that of other cancers. Surgery is an option; the removal of the tumor along with surrounding tissue may be vital for the patient's survival. For discrete, localized tumors, surgery is often followed by radiation therapy of the excised area to reduce the chance of recurrence. Radical surgical resection is the treatment of choice in MPNST. A good three-dimensional clearance is mandatory for a successful outcome. MPNST has the highest recurrence rate of any sarcomas, and adequate initial treatment gives the best chance of survival. ${ }^{[10]}$

\section{REFERENCES}

1. Gupta G, Maniker A. Malignant peripheral nerve sheath tumors. Neurosurg Focus 2007;22:E12.

2. Kim DH, Murovic JA, Tiel RL, Moes G, Kline DG. A series of 397 peripheral neural sheath tumors: 30-year experience at Louisiana State University Health Sciences Center. J Neurosurg 2005;102:246-55.

3. Stark AM, Buhl R, Hugo HH, Mehdorn HM. Malignant peripheral nerve sheath tumours - Report of 8 cases and review of the literature. Acta Neurochir (Wien) 2001;143:357-63.

4. Wanebo JE, Malik JM, VandenBerg SR, Wanebo HJ, Driesen N, Persing JA. Malignant peripheral nerve sheath tumors. A clinicopathologic study of 28 cases. Cancer 1993;71:1247-53.

5. Nambisan RN, Rao U, Moore R, Karakousis CP. Malignant soft tissue tumors of nerve sheath origin. J Surg Oncol 1984;25:268-72.

6. $X u$ Q, Xing $B$, Huang $X$, Wang $R$, Li $Y$, Yang Z. Primary malignant peripheral nerve sheath tumor of the cauda equina with metastasis to the brain in a child: Case report and literature review. Spine J 2012;12:e7-13.

7. Acharya R, Bhalla S, Sehgal AD. Malignant peripheral nerve sheath tumor of the cauda equina. Neurol Sci 2001;22:267-70.

8. Amin A, Saifuddin A, Flanagan A, Patterson D, Lehovsky J. Radiotherapy-induced malignant peripheral nerve sheath tumor of the cauda equina. Spine (Phila Pa 1976) 2004;29:E506-9.

9. Thomeer RT, Bots GT, van Dulken $H$, Luyendijk W, Helle P. Neurofibrosarcoma of the cauda equina. Case report. J Neurosurg 1981;54:409-11.

10. Yone K, Ijiri K, Hayashi K, Yokouchi M, Takenouchi T, Manago K, et al. Primary malignant peripheral nerve sheath tumor of the cauda equina in a child case report. Spinal Cord 2004;42:199-203.

11. Than KD, Ghori AK, Wang AC, Pandey AS. Metastatic malignant peripheral nerve sheath tumor of the cauda equina. J Clin Neurosci 2011;18:844-6.

12. Vogel KS, Klesse LJ, Velasco-Miguel S, Meyers K, Rushing EJ, Parada LF. Mouse tumor model for neurofibromatosis type 1. Science 1999;286:2176-9.

13. Zhou H, Coffin CM, Perkins SL, Tripp SR, Liew M, Viskochil DH. Malignant peripheral nerve sheath tumor: A comparison of grade, immunophenotype, and cell cycle/growth activation marker expression in sporadic and neurofibromatosis 1-related lesions. Am J Surg Pathol 2003;27:1337-45.

14. Ghosh BC, Ghosh L, Huvos AG, Fortner JG. Malignant schwannoma. A clinicopathologic study. Cancer 1973;31:184-90.

How to cite this article: Singh AS, Atam V, Das L, Patil M, Yathish $\mathrm{BE}$, Kunwar S, et al. Malignant primary nerve sheath tumor of the cauda equina in a patient without Von Recklinghausen disease. Indian J Neurosurg 2013;2:291-3.

Source of Support: Nil, Conflict of Interest: None declared. 